\title{
University of Michigan MHD results of the Geospace Global Circulation Model metrics challenge
}

\author{
A. J. Ridley, K. C. Hansen, G. Tóth, D. L. De Zeeuw, and T. I. Gombosi \\ Space Physics Research Laboratory, Department of Atmospheric, Oceanic, and Space Physics, \\ University of Michigan, Ann Arbor, Michigan, USA \\ K. G. Powell \\ W. M. Keck Foundation CFD Laboratory, Department of Aerospace Engineering, University of Michigan, \\ Ann Arbor, Michigan, USA \\ Received 7 August 2001; revised 21 December 2001; accepted 9 January 2002; published 11 October 2002. \\ [1] We present University of Michigan MHD code results of the "metrics challenge." We \\ have simulated the magnetospheric and ionospheric configuration during the 16-17 April \\ 1999 CME, the 10-11 December 1998 time period, and the 5-6 November 1998 time \\ period. The simulation results were compared to the DMSP cross-track velocities, and the \\ RMS differences were examined. We have found that the code reproduces the DMSP data \\ quite accurately except for the sharpness of the gradients and the transients which were \\ observed. The cross polar cap potential was well matched, as was the location of the \\ convection reversal boundary. In addition, the code reproduced features of statistical \\ models of the ionospheric electric potential. INDEX TERMS: 2740 Magnetospheric Physics: \\ Magnetospheric configuration and dynamics; 2753 Magnetospheric Physics: Numerical modeling; 2411 \\ Ionosphere: Electric fields (2712); 2463 Ionosphere: Plasma convection; KEYWORDS: magnetosphere, MHD, \\ ionosphere, metrics, convection, electrodynamics
}

Citation: Ridley, A. J., K. C. Hansen, G. Tóth, D. L. De Zeeuw, T. I. Gombosi, and K. G. Powell, University of Michigan MHD results of the Geospace Global Circulation Model metrics challenge, J. Geophys. Res., 107(A10), 1290, doi:10.1029/2001JA000253, 2002.

\section{Introduction}

[2] Validation of numerical models is accomplished in a number of stages. For example, there is need to verify that: (1) the codes are solving the equations correctly, (2) the codes converge to a given solution when the grid has increasingly better resolution, and (3) the model solution approximates what is observed in reality. While the first two validations are nontrivial, there is much less controversy about how to carry out these validations. The third type of validation is difficult because of the possible biases which can exist in the data selection and the methodology behind the data-model comparison. In addition, it is possible for models which have not been validated numerically to have errors which cancel each other out in certain circumstances, thereby giving the "correct" answer when compared with observations. It is therefore important to validate models in a number of different ways and over a broad range of physical conditions.

[3] With 3D MHD simulations, the validation is very difficult. There are very few, if any, 3D simulations which can be run and compared to analytic solutions. This makes it difficult to determine whether the codes are working correctly in the first place. Then, even basic features of the 3D magnetosphere are under debate. For example, the length of the tail during northward IMF is contested among the modeling community [Raeder, 1999, 2000; Gombosi et al., 2000].

Copyright 2002 by the American Geophysical Union. 0148-0227/02/2001JA000253
[4] The University of Michigan's magnetohydrodynamic (MHD) code (termed BATS-R-US) solves the MHD equations on a block-adaptive Cartesian grid [Powell et al., 1999]. This grid allows various scale sizes in the simulated region to be resolved. The code has been used to simulate comets, the heliosphere, Mercury, Venus, Mars, and the magnetospheres of Earth, Saturn, and Jupiter.

[5] Initial validation of the code was performed to show that the numerics are correct and that grid convergence is achieved [Powell et al., 1999]. They showed that the code matches analytic solutions of an oblique shock, and that the errors in density and temperature decrease linearly with increased resolution. Ridley et al. [2001] present an initial validation of the BATS-R-US code, in which ground-based magnetometer data was compared to simulated data from the MHD code. This initial validation was in response to the auroral electrojet challenge which focused on the 19-20 March 1999 time period. The MHD code was run to steady state for approximately average solar wind and interplanetary magnetic field (IMF) input conditions for those two days, and the results were compared for the entire 48 hour time period. These results showed that the MHD simulated magnetic perturbations were much lower than the measured values. This was explained as being possibly caused by the lack of region-2 currents and using a single steady state run to describe 48 hours of data.

[6] Other studies have been conducted where different MHD models have been compared to data. For example, Raeder et al. [1997] shows comparisons of a simulation 
with Geotail plasma and magnetic field observations in the tail. That study showed the satellite data in the global context of magnetosphere, which is one of the many uses of a MHD simulation. Raeder et al. [1998] shows ionospheric results from a MHD simulation compared with the results from the assimilative mapping of ionospheric electrodynamics (AMIE) technique [Richmond and Kamide, 1988]. They show that while the potential maps basically agree in shape, the MHD potentials are much larger than the AMIE potentials. Fedder et al. [1998] shows comparisons of a simulation result with DMSP data, similar to that described in this study. They show that their MHD code overpredicts the cross polar cap potential on a significant number of passes (24 of 28).

[7] In order to validate community models in a more consistent manner and quantify the differences between the simulations and geophysical measurements, several metrics were defined. The metric for the global MHD models is the root-mean-squared (RMS) difference between the ion flow measurements made by the Defense Meteorological Satellite Program (DMSP) satellites and those predicted by the MHD codes. The DMSP satellites are in Sun synchronous orbits at approximately $800 \mathrm{~km}$ altitude. The orbital period of the satellite is approximately 100 minutes. The ion drift sensor on board the satellites measures the horizontal and vertical velocity of ions perpendicular to the satellite track. Because of the offset of the geographic and magnetic poles, the DMSP satellites cover a large portion of the auroral and polar regions of both hemispheres. See Rich and Hairston [1994] and Hairston and Heelis [1995] for further general information on the DMSP measurements. The particular satellites used in this study include F13, in a dawn-dusk orbital plane, and F14, in a 09-21 LT orbital plane.

[8] The first "metrics challenge" included simulating 3 time periods covering a wide range of solar wind input conditions: (1) 16-17 April 1999, (2) 10-11 December 1998, and (3) 5-6 November 1998. The challenge was to simulate approximately 36 hours of ionospheric convection for each of these events and compare the results to the DMSP data. There were 181 total passes through all of these events with approximately 300 data points in each pass. For the first 2 events (April and December), the F14 satellite provided data for only the Northern Hemisphere, while the F13 satellite provided data for both hemispheres. For the November event, both satellites provided data in both hemispheres.

\section{Model}

[9] The BATS-R-US model is a 3D global MHD code which has the ability to simulate various plasma environments [Powell et al., 1999]. This model has been used to study the Earth's magnetosphere in a number of studies [Song et al., 1999; Groth et al., 2000]. BATS-R-US has a tilted, rotating dipole, with the rotation axis offset from dipole axis. The magnetospheric simulations are in GSM coordinates with the $X$ axis pointed toward the Sun and the dipole axis in the $X-Z$ plane. The corotation axis and dipole axis are usually specified in such a way that the axes match those in Earth's Northern Hemisphere.

[10] The MHD code has an inner boundary at $2.5 R_{e}$. At this inner boundary, the plasma density, temperature, and velocity are specified. In addition, the magnetic field is allowed to float, so currents can flow along the boundary. The velocities which are imposed on the boundary are calculated in the ionosphere in a three step process: (1) field-aligned currents are calculated from the curl of the magnetic field at $3.5 R_{e}$, and these are mapped down to the ionosphere, (2) a height-integrated ionospheric conductance pattern is generated (as described by Ridley et al. [2001]) and the ionospheric potential is calculated from the equation:

$$
j_{R}(R)_{e}=\left[\nabla_{\perp} \cdot(\Sigma \cdot \nabla \psi)_{\perp}\right]_{R=R_{e}}
$$

which describes the relationship between the height integrated conductance tensor $(\Sigma)$, the ionospheric potential $(\psi)$, and the radial component of the current $\left(j_{R}\right)$; and (3) the electric potential is mapped out along field lines to the 2.5 $R_{e}$ boundary, and electric fields and velocities are generated. The corotation velocity field is added to the ionosphere generated velocity field.

\subsection{Local Time Stepping}

[11] The BATS-R-US model has the ability to run in two different modes: (1) a time-accurate mode, similar to other MHD models and (2) an iterative local time stepping mode in which each cell takes different time steps. The local time stepping mode rapidly converges to the steady state solution through unphysical intermediate states. In this mode, the upstream boundary condition (i.e. the IMF and solar wind) is held constant, and a steady state magnetospheric configuration is derived for those upstream conditions and the given dipole and corotation tilt. While the iterations leading up to the steady state configuration are nonphysical, once the $d / d t$ terms decrease significantly, the magnetospheric configuration can be interpreted. This is usually accomplished in approximately 7500 iterations. The main disadvantage of running the code in the local time stepping mode is that there is no history to the solution, which is obviously wrong in the case of the magnetosphere. When examining the directly driven portion of the magnetosphere, though, such as the dayside flow patterns and current systems for given IMF orientations, this method works exceptionally well.

[12] In the more common time-accurate mode, the code is run with each cell taking the same time step. To drive the model toward steady state, the IMF must be swept through the entire simulation domain $\left(256 R_{e}\right)$ at the solar wind speed (approximately $400 \mathrm{~km} / \mathrm{s}$ ) using the approximately 0.05 second time step. Using these approximations, the IMF would propagate across the simulation in 1.13 hours, or 82000 iterations. This time period is simply to transport the IMF to the entire simulation domain, while the magnetosphere does not reach a steady state until approximately twice the length of time. For simulations of time-varying phenomena, such as IMF turnings, or solar wind pressure jumps, the code is driven to a steady state using the local time stepping with the initial IMF and solar wind conditions and then used in a time-accurate mode for the rest of the run.

[13] For the metrics challenge events, we chose to run the code to steady state using local time stepping and instantaneous IMF and solar wind conditions. This allowed the use of much higher resolution in the inner magnetosphere. The IMF during some of the events was rotating slowly enough that a series of steady state snapshots was an adequate 

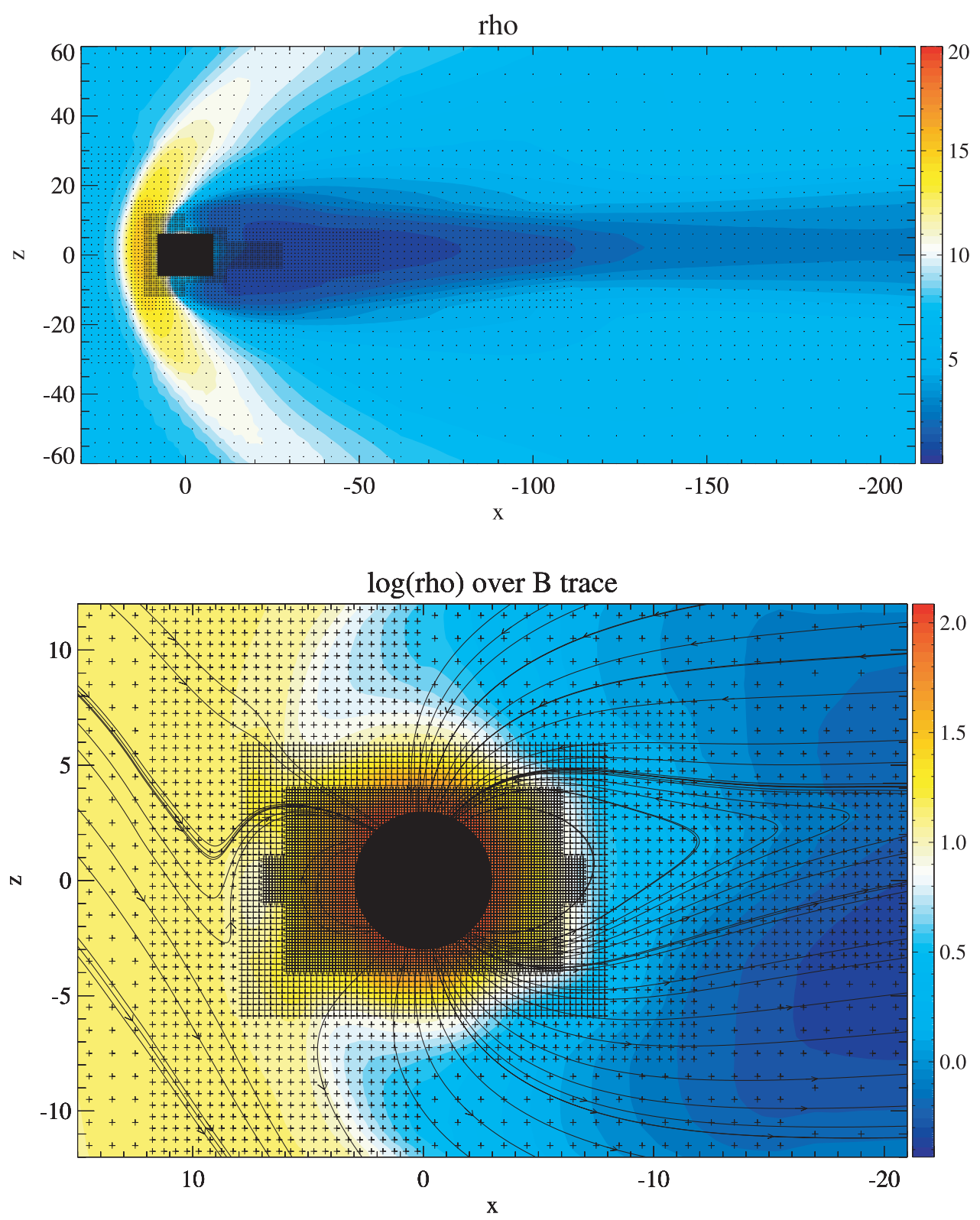

Figure 1. The top figure shows almost the complete simulation domain in the $X-Z$ plane with the grid points shown as dots. The largest spacing between cell centers is $8 R_{e}$. The color contours show the number density in particles/cc. The lower plot shows the region near the inner boundary (at $\left.3.0 R_{e}\right)$. The highest resolution used in these simulations, and shown in the plot, is $0.125 R_{e}$. This plot shows the log of the number density as a color contour with the magnetic field lines traced out. The slight tilt in the dipole is evidenced by the kinking of the field lines in the tail occurring above the equatorial plane.

representation of the magnetosphere. For the April 1999 event, we ran the model in both modes to compare the results.

\subsection{Grid Structure}

[14] Figure 1 shows the grid structure of the simulations. The domain included the space incorporated between $x=32$ and -224 and $y, z= \pm 64$. The grid resolution ranged from 8 $R_{e}$ downtail to $1 / 8 R_{e}$ in the inner magnetosphere. Although the code is capable of adapting the grid to resolve regions of importance better, the grid was kept constant between all of the runs. There were approximately 800,000 cells in the simulation with most concentrated within the inner magne- tosphere. For the time-accurate simulation, the finest resolution was decreased to $1 / 4 R_{e}$, which required about 300,000 cells.

\subsection{Model Tuning}

[15] The "rules" of the challenge specified that the any tuning of the model must be done such that it is not event specific, but is generalized so all events are run with the same code. The ionospheric boundary in the simulations have therefore undergone revisions since the study of Ridley et al. [2001]. While the overall structure of the ionosphere is the same, small modifications have been added to better represent the observed phenomena. The ionosphere was 
Table 1. RMS Errors of the Tuning Runs Computed Using the Derivative of the Potential From the MHD Model and the DMSP Data, Instead of the True Winds

\begin{tabular}{lcccccc}
\hline Time & Hemisphere & DMSP & ${\text { Run } 1^{\mathrm{a}}}$ & ${\text { Run } 2^{\mathrm{b}}}^{\mathrm{R}}$ & Run $3^{\mathrm{c}}$ & ${\text { Run } 4^{\mathrm{d}}}^{\mathrm{n}}$ \\
\hline 1319 & North & 0.736 & 0.516 & 0.520 & 0.520 & 0.509 \\
1409 & South & 0.682 & 1.092 & 0.845 & 0.810 & 0.754 \\
1524 & South & 0.526 & 0.972 & 0.674 & 0.581 & 0.551 \\
1641 & North & 0.450 & 0.310 & 0.332 & 0.335 & 0.336 \\
1822 & North & 0.400 & 0.354 & 0.374 & 0.381 & 0.383 \\
1844 & South & 0.350 & 0.500 & 0.374 & 0.361 & 0.355 \\
Average & both & 0.524 & 0.624 & 0.520 & 0.498 & 0.481 \\
\hline
\end{tabular}

${ }^{a}$ Polar cap Pedersen conductance uniform 0.25 mhos, no seasonal dependence.

${ }^{\mathrm{b}}$ Polar cap Pedersen conductance uniform 7.5 mhos, seasonal dependence on strength of auroral oval: $\zeta=0.75 \cos \left[-\left(\Theta / \Theta_{\max }\right) \pi / 2.0+\pi / 2.0\right]+$ 1.50 , where $\Theta$ is the dipole tilt. At highest dipole tilt (winter), the oval conductance is increased by $225 \%$. At lowest dipole tilt (summer), the oval conductance is decreased by $25 \%$.

${ }^{\mathrm{c}}$ Polar cap Pedersen conductance uniform with seasonal dependence $\left(\Sigma_{P e d}=\zeta^{2.5}\right.$, where $\Sigma_{P e d}^{P C}$ is the Pedersen conductance). The auroral oval was the same as Run 2.

${ }^{\mathrm{d}} \Sigma_{P e d}^{P C}=-3.75 \cos [(\zeta-0.75) \pi / 1.5]+4.0$. The auroral oval had the same seasonal dependence as in Runs 2 and 3, but the field-aligned currents were smoothed as described in the text.

"tuned" using 6 DMSP passes in the April 1999 time period. The April time period was chosen because it was consider the most important time period in the challenge. Some of these 6 passes were badly modeled, while some were modeled well. The goal of the tuning was to significantly reduce the RMS error of the "bad" passes, while not increasing the RMS error of the "good" passes. Table 1 describes the RMS errors which were achieved before and after the tuning. The modifications to the code include:

1. An artificial region 2 field-aligned current (FAC) system was added. This was modeled as a simple sin function with a maximum (upward current) at dawn and a minimum (downward current) at dusk. The strength of the maximum and minimum are 0.75 times the maximum region $1 \mathrm{FAC}$. The width of the system is approximately $5^{\circ}$ in latitude and is located near the equatorward edge of the region 1 current system. These currents were added for 2 reasons: (1) to decrease the penetration of the potential to lower latitudes, which causes the electric field and velocity to be sharper near the equatorward edge of the pattern; and (2) to reduce the cross polar cap potential by allowing some of the region-1 current to flow equatorward instead of across to pole. Most fully consistent ideal-MHD models of the magnetosphere are not able to produce large region-2 current systems for a number of reasons, which may include: grid resolution, inner boundary location and values, location and strength of the reconnection region in the tail, and possibly the temperature and density of the plasma sheet. A detailed discussion of this is beyond the scope of the current study and will be address in later studies.

2. Because the relationship between the FAC and the particle precipitation in the code is done on a grid point by grid point bases, regions between the upward and downward FAC (i.e. 0 FAC), had little particle precipitation. To improve this, a $10^{\circ}$ latitudinal average is done on the absolute value of the FAC, and then the relationship between the localized FAC and the particle precipitation is used. This makes the aurora smoother with less of a double oval structure.
3. The dipole tilt in the MHD code is dependent both on the day of the year and the time of day, such that at approximately $1645 \mathrm{UT}$ on any given day, the dipole is tilted toward the sun to it's furthest extent. Therefore at certain times of the day, there is a strong asymmetry in the dayside ionospheric conductance between the two hemispheres. With similar FAC magnitudes into each hemisphere, this can result in very different electric fields, with the "winter" hemisphere having significantly stronger fields. This was not observed in the April, 1999 DMSP data (although it was observed in the simulation results), so a dipole-tilt dependence on the particle precipitation in the main oval and the polar cap was implemented. The polar cap Pedersen conductance is tuned such that at the furthest "summer" tilts, the conductance is 0.25 mhos, while at furthest "winter" tilts, the conductance is 7.5 mhos. The polar cap is defined as the area poleward of peak auroral precipitation. The conductance specified in this region was greater than or equal to those values specified above. For the main oval the multiplication factor in the particle fluxes ranged from 0.75 to 2.25 . See Table 1 for the exact functional form of the multiplication factors. While these values may be overly large, Liou et al. [1997] showed that the UVI brightness does have a seasonal dependence, with the brightest times occurring during the winter season.

[16] Table 1 shows that the above changes significantly lowered the RMS differences between the DMSP data and the ionospheric convection simulated by the MHD code. Because of the rules of the metrics challenge specified that the tuning must be conducted before modeling of all of the events, the above changes were implemented before the runs were conducted.

[17] It is interesting to note that the model tuning improved the results for the April time period, but tended to increase the RMS error in the November and December periods, as will be discussed below.

\section{Model Results}

\subsection{April 1999 Event}

[18] During 16-17 April 1999, the solar wind and IMF were consistent with a coronal mass ejection. Figure 2 shows that during 17 April, the nearly constant strength magnetic field was slowly rotating, with a strong density enhancement as a precursor for the unusually strong field. The first passes by the DMSP satellites included in the metrics challenge were around $1800 \mathrm{UT}$, which was just after the $B_{x}$ turned negative. The passes continued until the end of 17 April.

[19] Because of the slow rotation in the IMF, it was decided to run the code in two modes - a local time stepping mode, and a time-accurate mode. The time-accurate simulation was run using the solar wind data shown in Figure 2 as the time-dependent upstream boundary condition. The side boundaries were far enough away from the magnetosphere and had zero normal gradient, so they had little influence on the solution. The data in Figure 2 was delayed by 1 hour to allow for the propagation between the $\mathrm{L} 1$ point and the upstream boundary. While this value may be quite inaccurate, the study by Ridley [2000] showed that the time delay error in any propagation could be off by many 

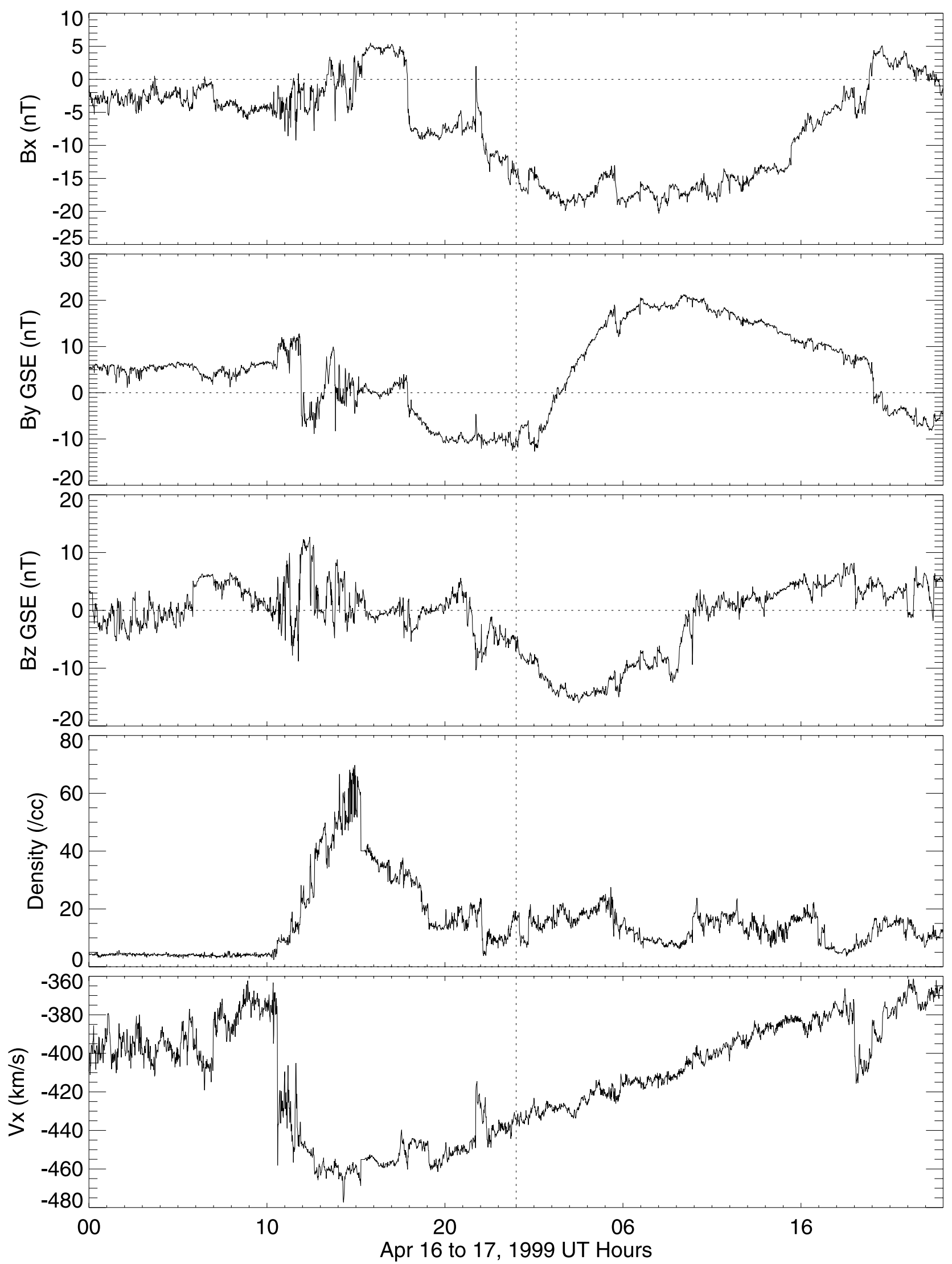

Figure 2. The IMF and solar wind parameters which were used as input into the simulation for the April 1999 event. Two days are shown, with the day demarcation shown as a dotted vertical line. The $V_{y}$ and $V_{z}$ data are not shown, but are small compared to the $V_{x}$ component. The code automatically rotates the input $\mathbf{B}$ and $\mathbf{V}$ from GSE to GSM coordinates. 

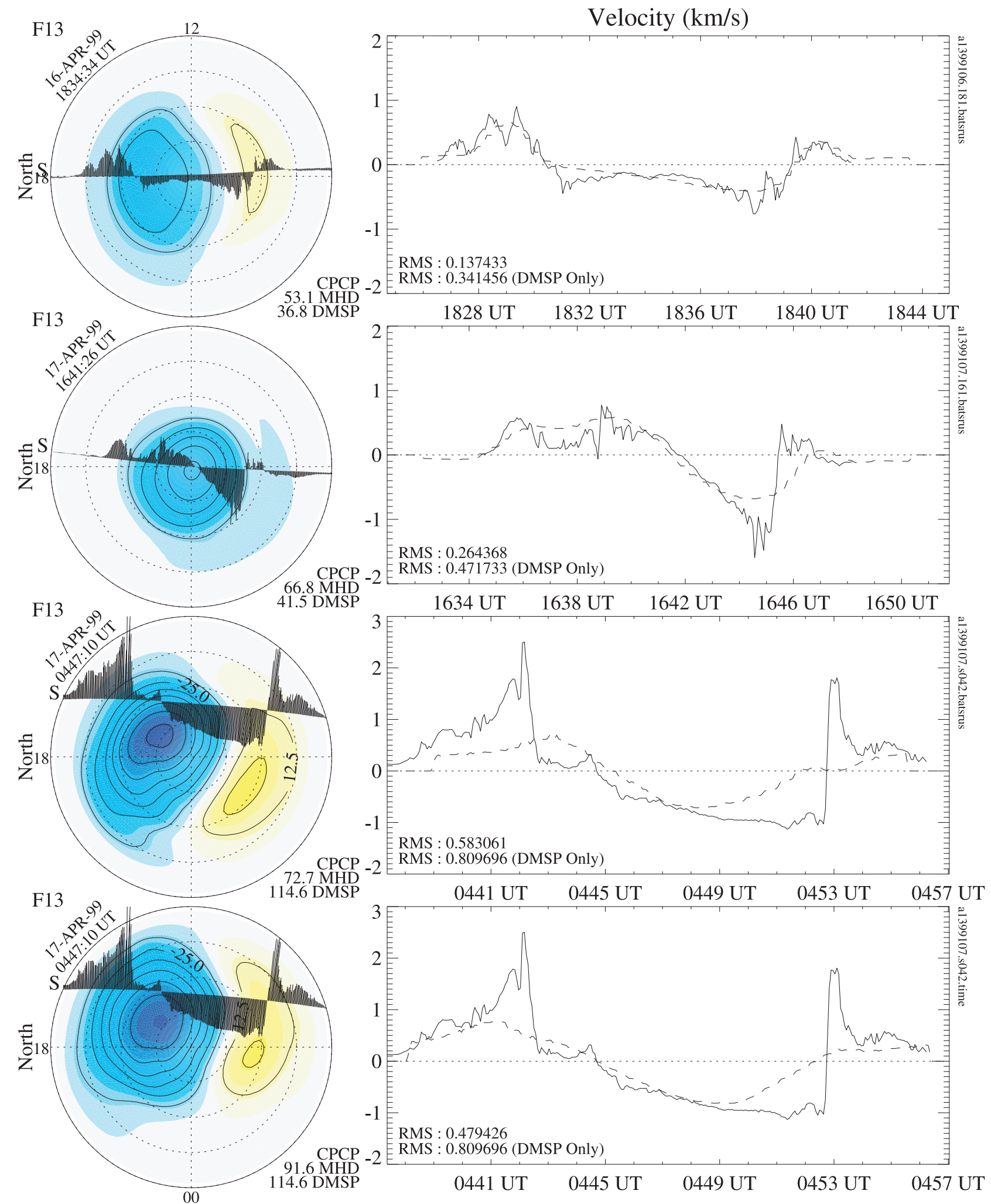

Figure 3. The panels to the left show the MHD results at the given times with the DMSP pass over plotted. The time corresponds to the time at which the DMSP satellite was closest to the pole. The cross polar cap potential (CPCP) measured by DMSP and the MHD code (along the satellite track) are given. The contours are $12.5 \mathrm{kV}$. The start of the DMSP pass is indicated by a " $\mathrm{S}$ " just outside of the plot. The hemisphere plotted is indicated on the far right. Each plot shows noon at the top and dawn to the right, with the lowest latitude plotted being $50^{\circ}$. The plots to the right show the measured and modeled crosstrack ion flow velocities. The root-mean-squared (RMS) differences between the two are indicated, the RMS variation in the data is further indicated. 
Jy over B trace
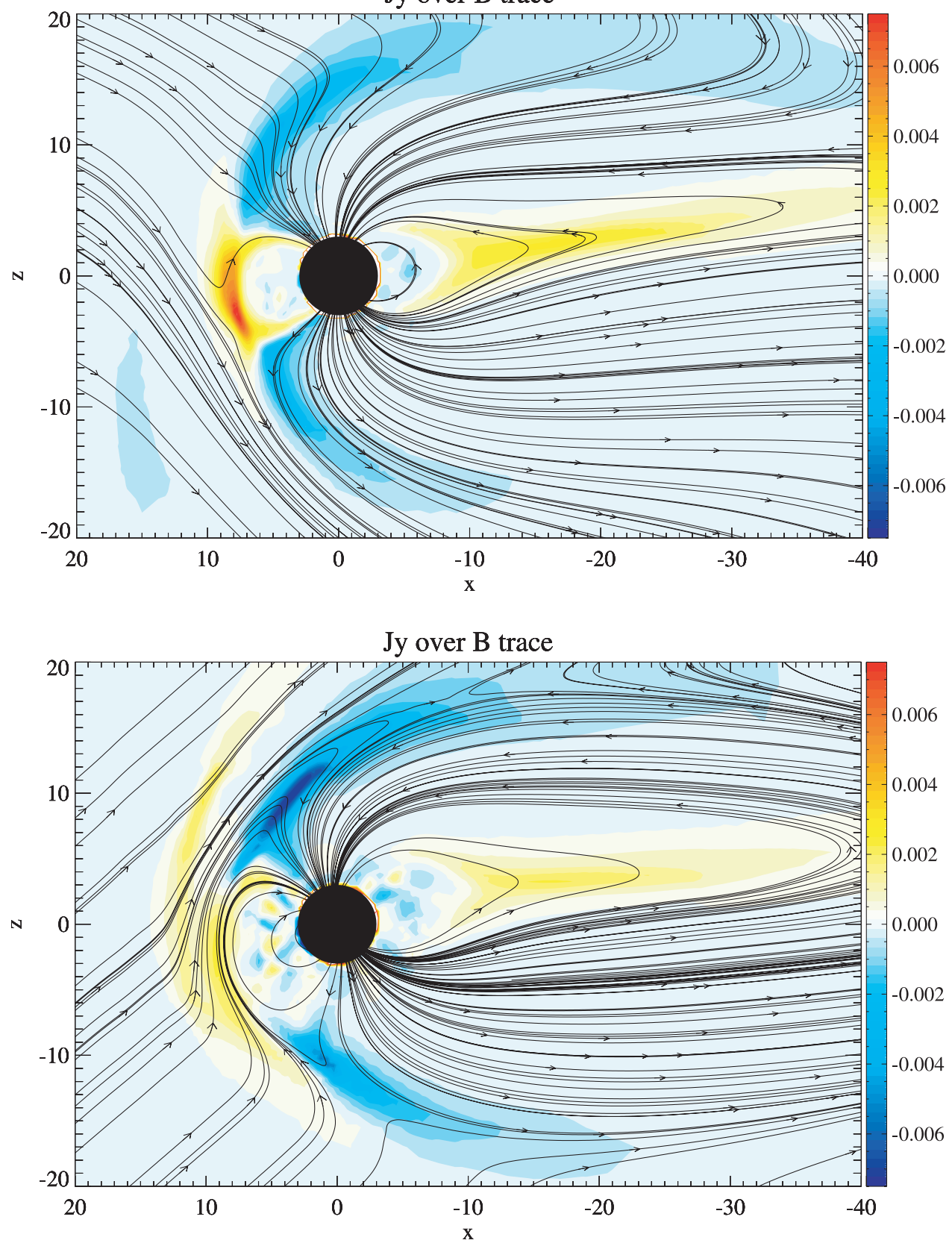

Figure 4. These figures show a noon-midnight cut of the magnetosphere at 2 time periods during the April 1999 event. The top panel shows the magnetosphere at April 17, 0954 UT, while the bottom plot shows it at 1641 UT. Each plot shows the magnetic field line traces over the current into and out of the page (i.e. $J_{y}$ ). These figures show how the dipole tilt changes as a function of UT during the day, and it shows how dramatically the current systems and magnetic field can change for different IMF orientations.

minutes. In addition, the variations occurred so slowly that errors under a few minutes do not contribute significantly to errors in the model results. For the local time stepping mode, a steady state solution was derived for each of the passes, where the IMF data used was the instantaneous value one hour before the DMSP pass reached its highest latitude.

[20] Figure 3 shows the ionospheric solution for 3 of the passes during the 16-17 April time period with the DMSP data plotted over the model result. The plots to the right show the measured cross-track ion velocities and the modeled values. The first 3 plots show model results for steady state solutions, while the last plot shows the same time as the 3rd plot, but it is a time-accurate solution. The DMSP data has much more variability than the simulation. Because of the nature of satellite measurements, it is impossible to tell whether this variability is temporal or spatial in nature. The DMSP data also has a higher maxima and minima than the model results, although this did not always imply that the cross polar cap potential measured by DMSP was larger. This is because the cross polar cap potential is an integral of the electric fields. If the electric fields have a lot of spikes are wildly varying, the integral can be lower than if the 

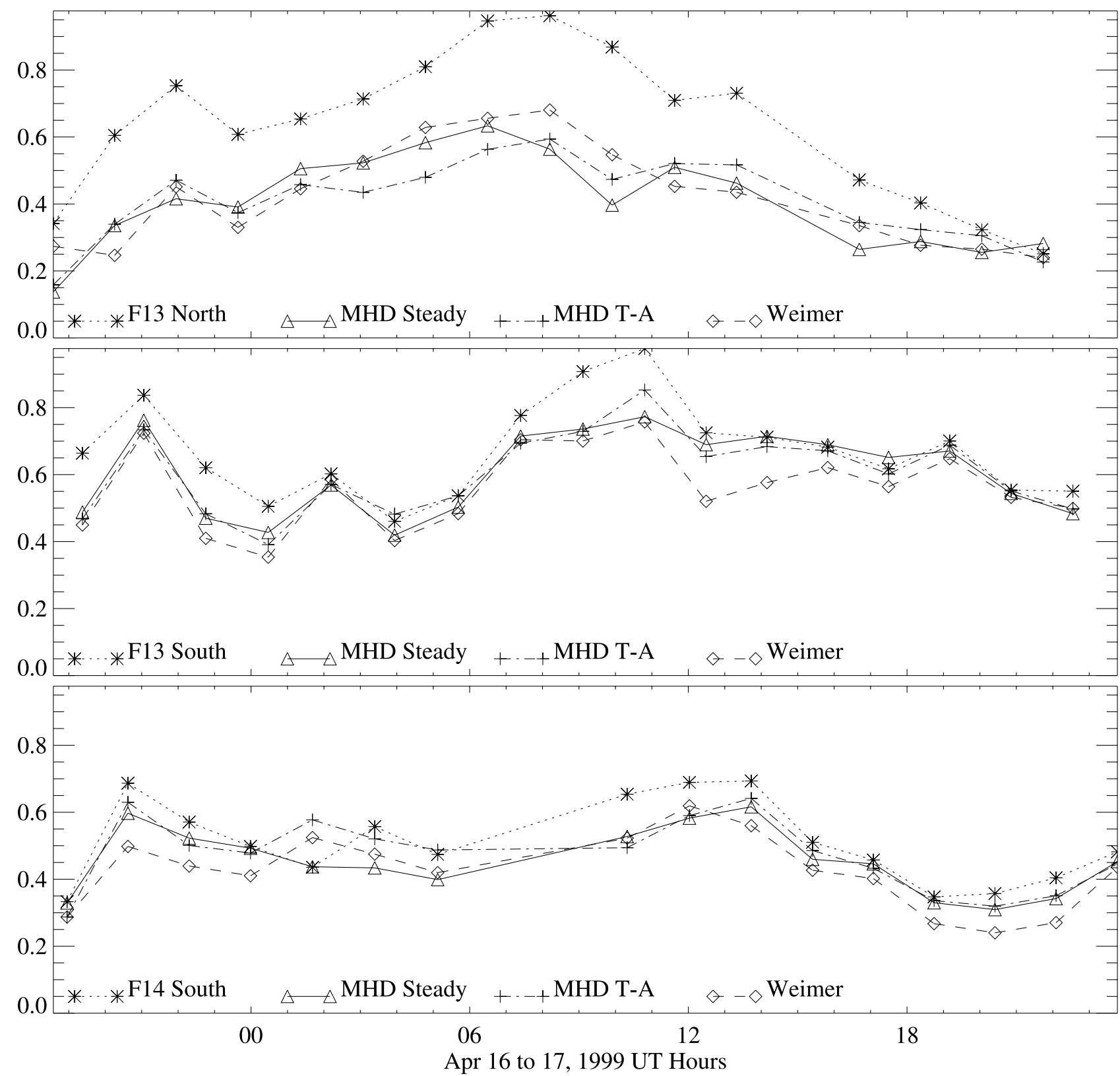

Figure 5. The RMS error in the various model runs of 16-17 April 1999. The dotted line shows the RMS variation in the DMSP data. The top two plots show the F13 satellite passes (north on top, south below), while the bottom plot shows the F14 passes (South only).

electric fields were smaller, but more regular. For most of the passes, the locations of the flow reversals were well predicted by the simulations, however the DMSP reversals were typically much sharper than those predicted by the model. This may be the result of a number of factors. For example, the strong shear may be caused by the extreme closeness of the region 1 and region 2 current systems. Because of the ad hoc region 2 currents described above, the currents may have been spaced further apart than reality. This would give a gradual change in the velocity as opposed to the sharp gradient observed in the data. A second source of error may arise from getting the conductance in the wrong location. For example, in the regions in which the DMSP data show strong flows, there should be little conductance, while in regions of little flow there may be large conductances. In reality these regions may be very close together, while in the MHD code, the conductance is much more smeared, resulting in lower flows in most locations and very few sharp gradients.

[21] For reference, plots of two magnetospheric solutions are show in Figure 4. These plots demonstrate the tilted dipole effects of the location of the merging region, and the direction of the tail. The top plot is during a time period when the dipole tilt is close to the rotation tilt in the $X-Z$ plane, while the lower plot shows the simulation a few hours later, when the dipole tilt is much larger, due to the dipole tilt offset from the rotation axis.

[22] Figure 5 shows the RMS errors for the different simulations during the April time period. Along with these are the Weimer [1996] results and the DMSP RMS varia- 

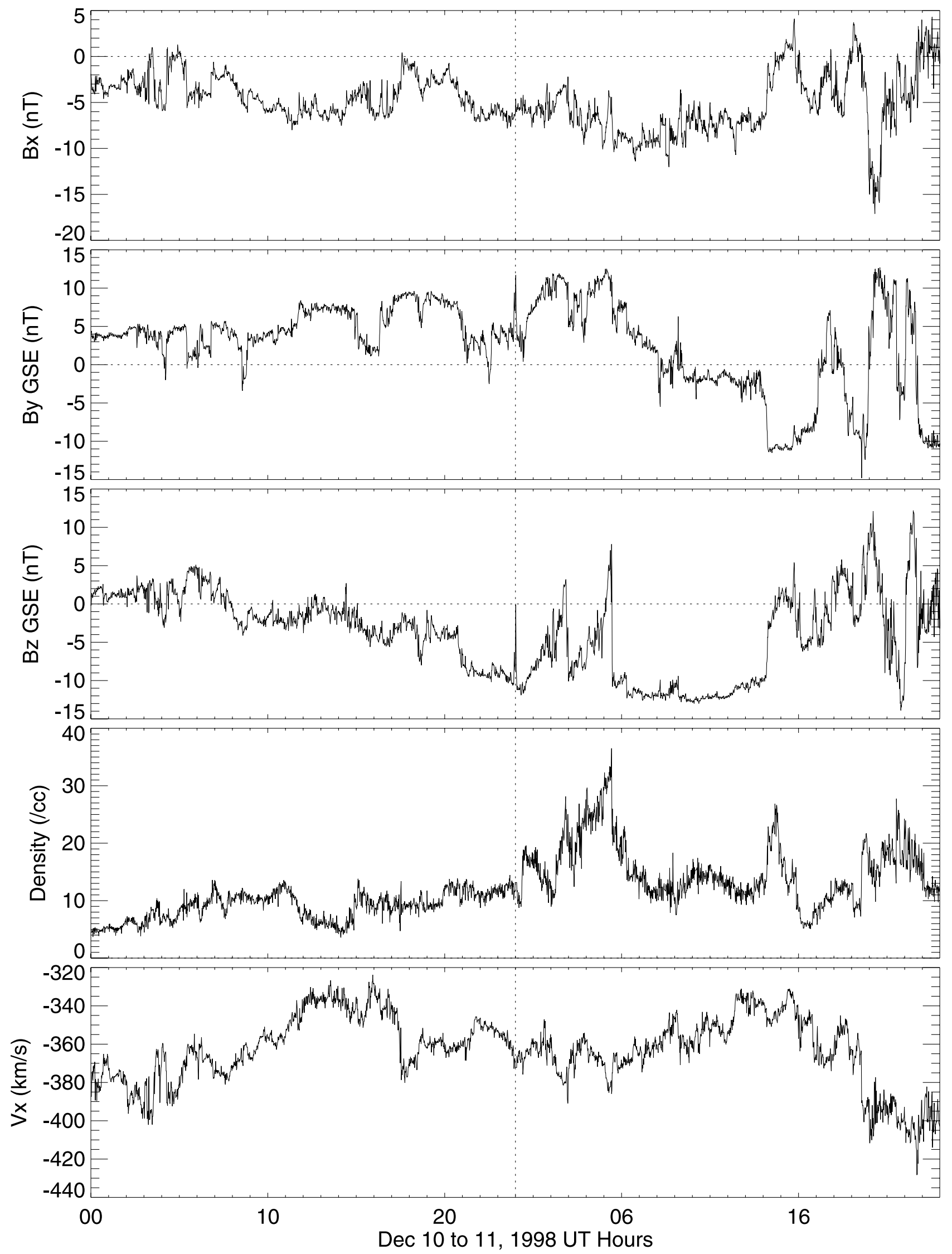

Figure 6. The IMF and solar wind parameters which were used as input into the simulation for the December 1999 event in the same format as Figure 2. 

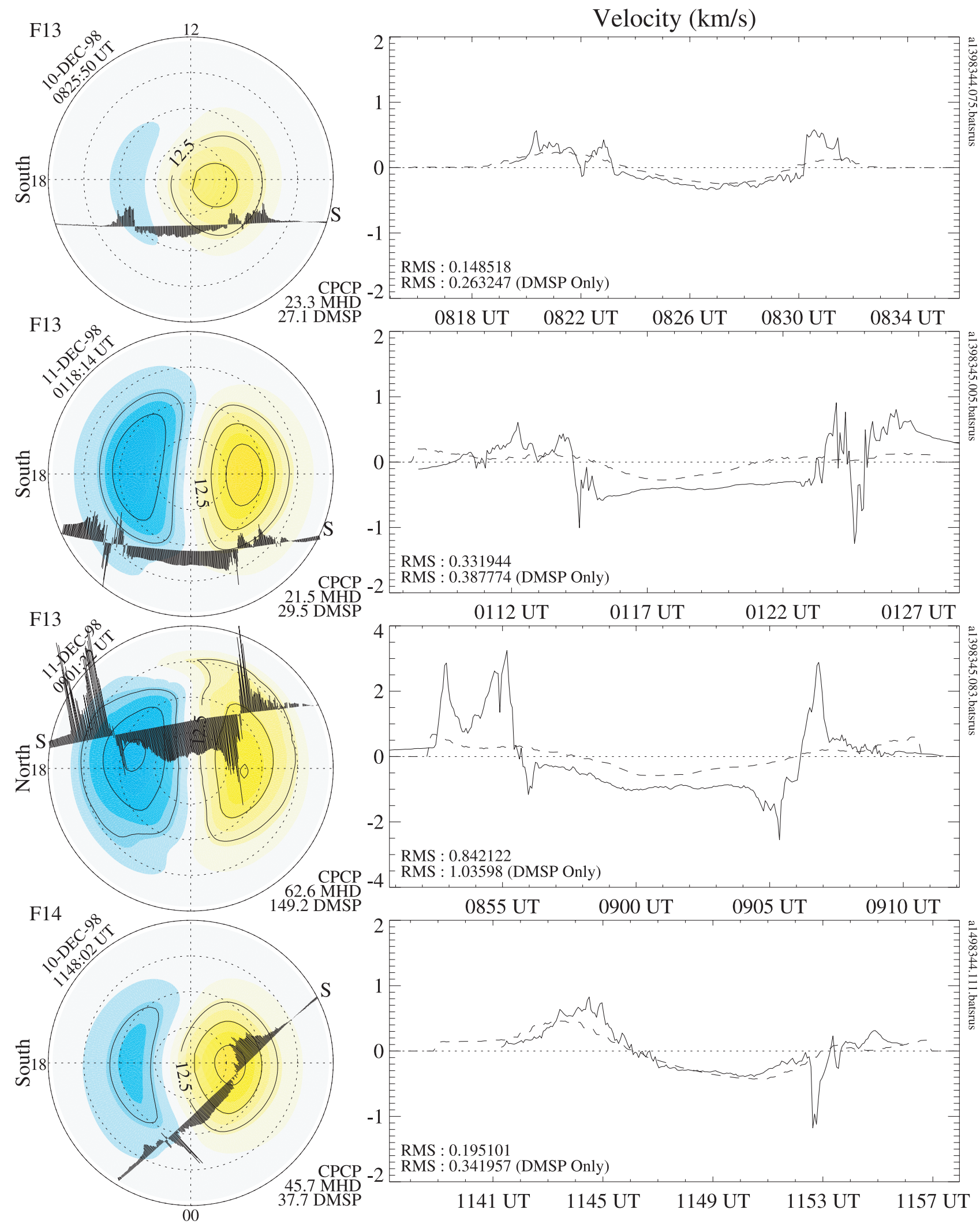

Figure 7. This figure shows two of the best and two of the worst comparisons between the DMSP data and the MHD results for the December time period in the same format as Figure 3. 

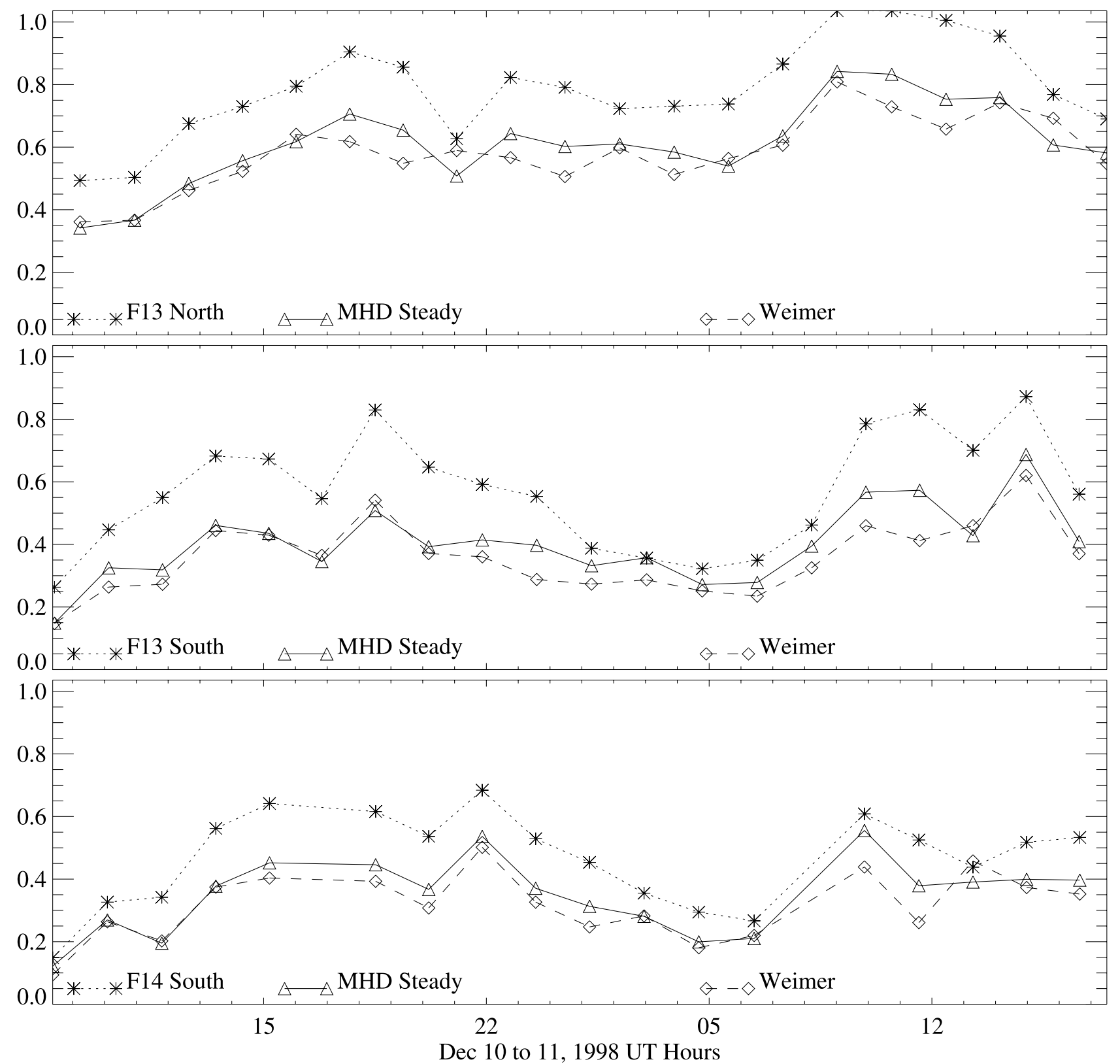

Figure 8. The RMS error for 10-11 December 1998, in the same format as Figure 5.

tion. The Weimer [1996] results show how one of the top of the line statistical models would fair using the exact same inputs as the steady state MHD simulations. The goal for the simulation community is to match or beat the Weimer-like models, which match the general trends in data, using first principles based simulations.

[23] The DMSP variation is defined as the RMS difference between the DMSP cross-track velocity and zero velocity. This basically quantifies the level of activity measured by the satellite. In addition, it shows that if the different RMS errors are less than this value, then the simulations are showing that the flows are generally in the same direction as the DMSP flows. If the values are larger, then the flow speeds may be in a different direction than the DMSP data shows.
[24] Returning to Figure 5, in the Northern Hemisphere, the model RMS error is significantly lower than the DMSP variation. This shows that in general the models were reproducing the general trends in the DMSP data during this time. The ratio between the RMS error and the DMSP variation (in the Northern Hemisphere) starts to increase after approximately $12 \mathrm{UT}$, which is the time in which $B_{z}$ started to become large and positive. In the Southern Hemisphere, the RMS variation and the RMS error are very similar in magnitude. In the Northern Hemisphere, the Weimer [1996] and simulation RMS errors were very similar throughout the entire interval. The Southern Hemisphere flows were much more unstructured and were therefore much more difficult to model. During the northward period (after 12 UT), the Weimer [1996] patterns clearly had less RMS error than the 
simulations. During the time period before this, though, the Weimer [1996] patterns where much closer (in RMS error) to the simulated patterns.

[25] It is interesting to note that the steady state model results were mostly better than the time-accurate results. As one would expect, the time-dependent runs appeared to do better during periods in which the IMF was varying, such as the large rotation in $B_{y}$ between 0200 and 0500 UT on 17 April. For example, Figure 3 shows a time-accurate solution (4th plot) compared to a local time stepping solution during this time period. The time-accurate result is better in every way than the local time stepping run: the cross polar cap potential is more consistent, the convection reversal boundaries are more consistent, the location of the maximum flow velocity is closer, and therefore the RMS is significantly lower. The local time stepping results were better in most cases because of the increased resolution of the simulation (1/8 $R_{e}$ as the smallest cell size, as opposed to $1 / 4 R_{e}$ for the time-accurate simulation).

\subsection{December 1998 Event}

[26] For this event, the MHD code was run in a local time stepping mode only. The DMSP passes started at approximately 0800 UT on the 10 th and continued until 1800 UT on the 11th. Figure 6 shows the IMF and solar wind conditions for the 10-11 December 1998 time period. This interval had periods in which $B_{z}$ was relatively low, such as between 0600 and 1400 UT on December 11th. One would therefore expect very large flows in the MHD code during this time period. This was not the case.

[27] As explained above, the tuning in the first event forced more conductance in the winter hemisphere, thereby suppressing the large electric fields. Unlike the April time period, the DMSP satellite measured larger flows in the winter hemisphere (the Northern Hemisphere) than in the summer hemisphere. Therefore, the MHD results were not qualitatively consistent with the DMSP data. Figure 7 shows examples of this behavior. The middle two plots show that the simulated flows are much too small, while the simulated CPCP values are much weaker than they should be.

[28] Surprisingly, though, the RMS errors (Figure 8) are quantitatively comparable to (or even better than) the April event. This is because the MHD code ended up getting the general flow structure correctly, but mostly underestimated the magnitude of the flow. In the April event, the structure in the DMSP data was very difficult to reproduce, so RMS was high. A flaw in using the RMS error as a proxy for how well a code is doing is that the error does not illuminate whether there is a systematic error in the comparison (such as being consistently low, as in the December case), or whether the error are entirely random (which is more like the April case).

[29] When the RMS errors of the simulation are compared to the RMS errors in the Weimer [1996] model, it is more evident that there is a problem with the simulation. The statistical model does consistently better for almost all of the passes. This is because the Weimer [1996] patterns are getting the flow speeds correct, while the MHD code consistently underestimates them.

[30] Figure 8 also illuminates that there is a hemispheric difference in the results: The Northern Hemisphere errors are much larger than the Southern Hemisphere. While the
DMSP variations are much larger in the Northern Hemisphere also, the average ratio of the RMS error to the DMSP variation is actually larger in the Northern Hemisphere: 0.779 as opposed to 0.723 in the Southern Hemisphere. This implies that the Northern Hemisphere solution is worse than the Southern Hemisphere. These factors indicate that the conductance added to the winter hemisphere may be far too much, and that the tuning may need to be changed to reduce this.

\subsection{November 1998 Event}

[31] Figure 9 shows the IMF and solar wind data over 5-6 November 1998. The DMSP passes started at approximately 1130 UT on the 5th and continued until 1900 UT on the 6th. The IMF is constantly changing, although it is consistently $B_{y}$ dominated except for a small time around 06 UT on the 6 th.

[32] Figure 10 shows four time periods during the November interval, with the top and bottom panels being some of the best comparisons, while the middle two panels are two of the worst. This is an interesting time period because a number of the DMSP passes do not appear to match the corresponding IMF values. For example, at 2036 UT (the second plot in Figure 10), the DMSP measured convection is has an elongated region of antisunward flow surrounded by regions of roughly equal sized sunward flow. This is indicative of a strong southward IMF, while the IMF shown in Figure 9 indicates that $B_{y}$ was strongly dominant during that time. The IMF shown in this figure are in GSE coordinates, so there is a small rotation to put them into the correct GSM coordinate system, but even with this rotation, $B_{y}$ is still dominate by many nT. Another example is the 0927 pass, in which there is clearly a large flow pattern which is not simulated. This time, the main problem could be related to using the local time stepping mode instead of a time-accurate simulation. At approximately $0830, B_{z}$ was changing, with a strong northward perturbation around this time. The magnetosphere may not have reacted strongly to this northward IMF, while the simulation time selected was almost exactly during the peak of the northward IMF. A time-accurate run may have had a much stronger convection during this time period.

[33] Because of the large oscillations in IMF, the Weimer [1996] model did not perform well either, as indicated in Figure 11. For this entire period, the MHD and Weimer [1996] results were very consistent with each other. They each stayed under the RMS variation of the DMSP data for the most part, although the results are much closer to the RMS variation than the December event and the Northern Hemisphere results for the April event.

[34] This is most likely due to the highly structured IMF. Because we used steady state solutions based on the instantaneous IMF values, there could be a many physical processes which are missed by the simulation, such as substorms and bow shock filtering of the incoming small scale structure of the IMF. These processes may alter the ionospheric convection significantly.

\section{Discussion}

[35] The validation presented by Ridley et al. [2001] showed that the MHD code reproduced the currents on 

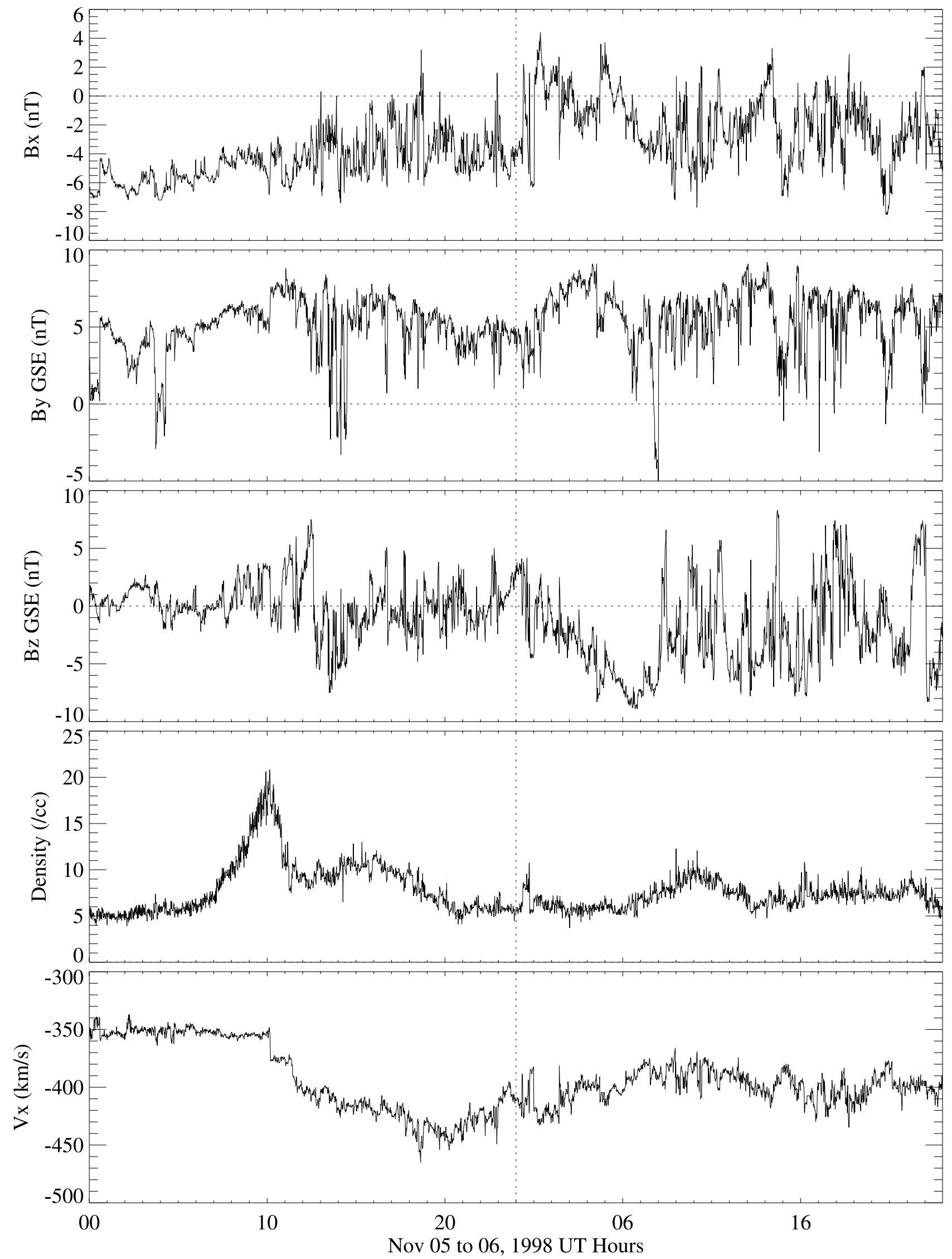

Figure 9. The IMF and solar wind parameters which were used as input into the simulation for the November 1999 event in the same format as Figure 2. 

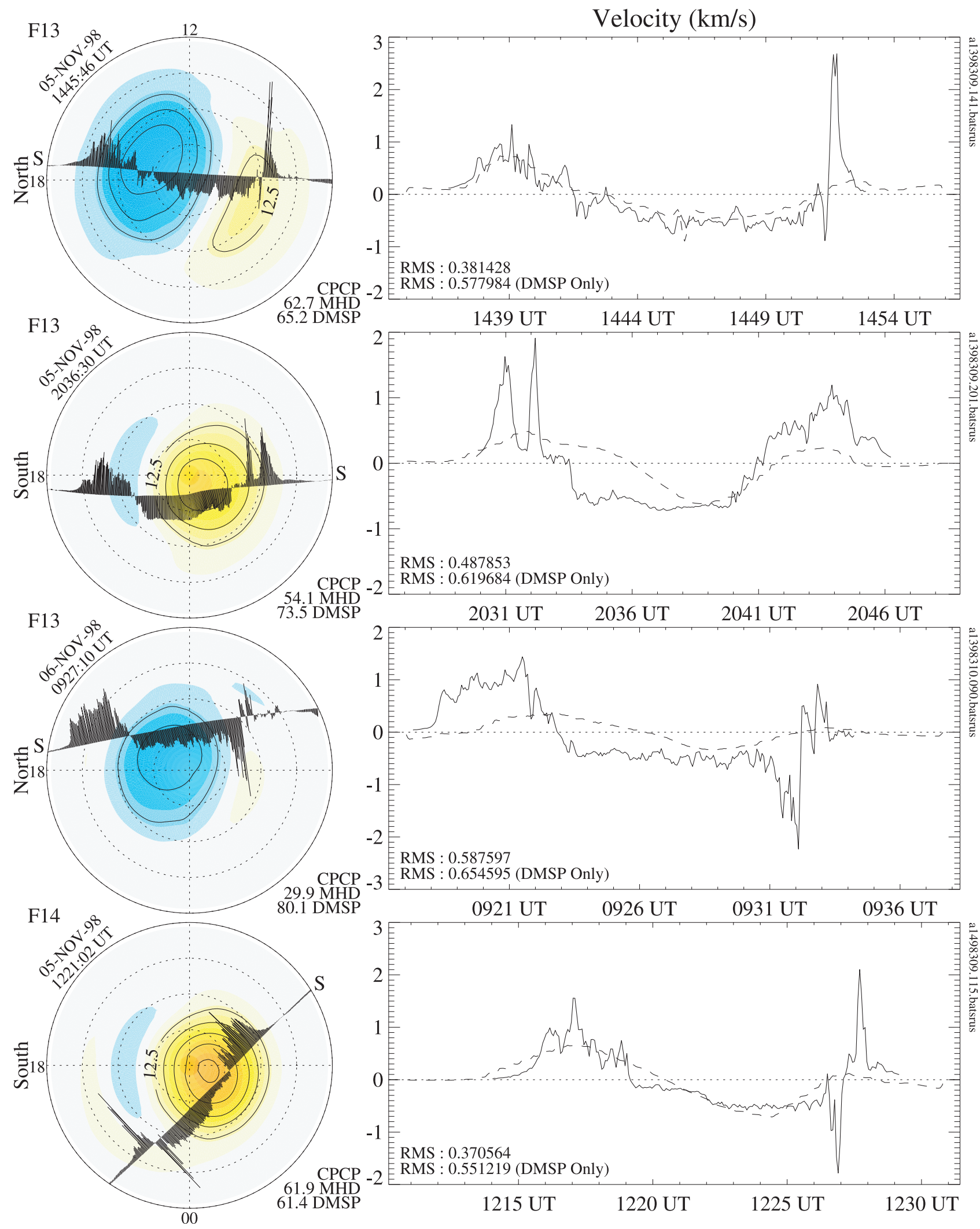

Figure 10. This plate shows the best comparisons between the DMSP data and the MHD results for the December time period in the same format as Figure 3. 

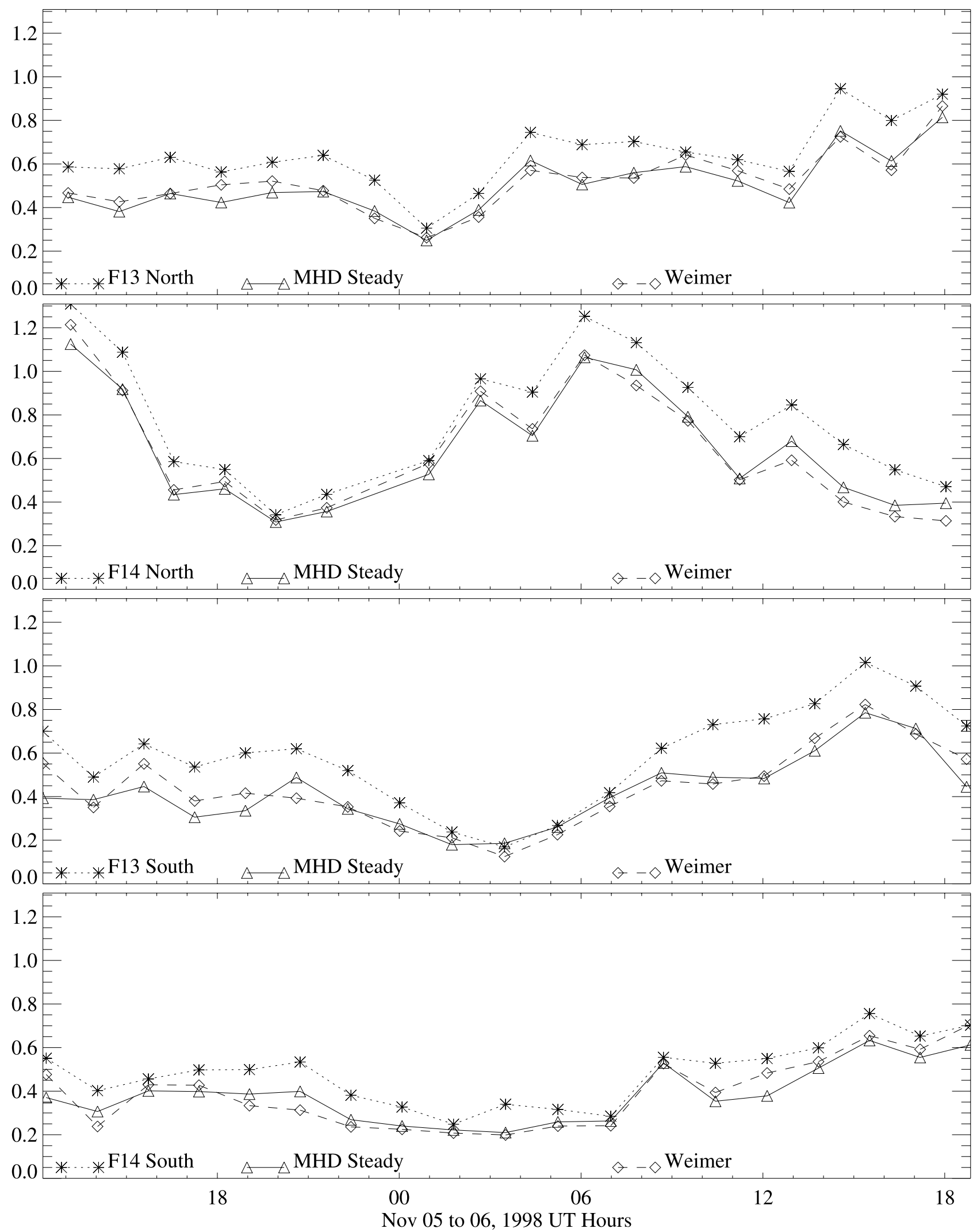

Figure 11. The RMS error for 5-6 November 1998 in the same format as Figure 5. The F14 satellite was reporting data in both hemispheres for this event, so the top two plots are Northern Hemisphere (F13 on top), while the bottom two are Southern Hemisphere (F13 on top). 
MHD Only
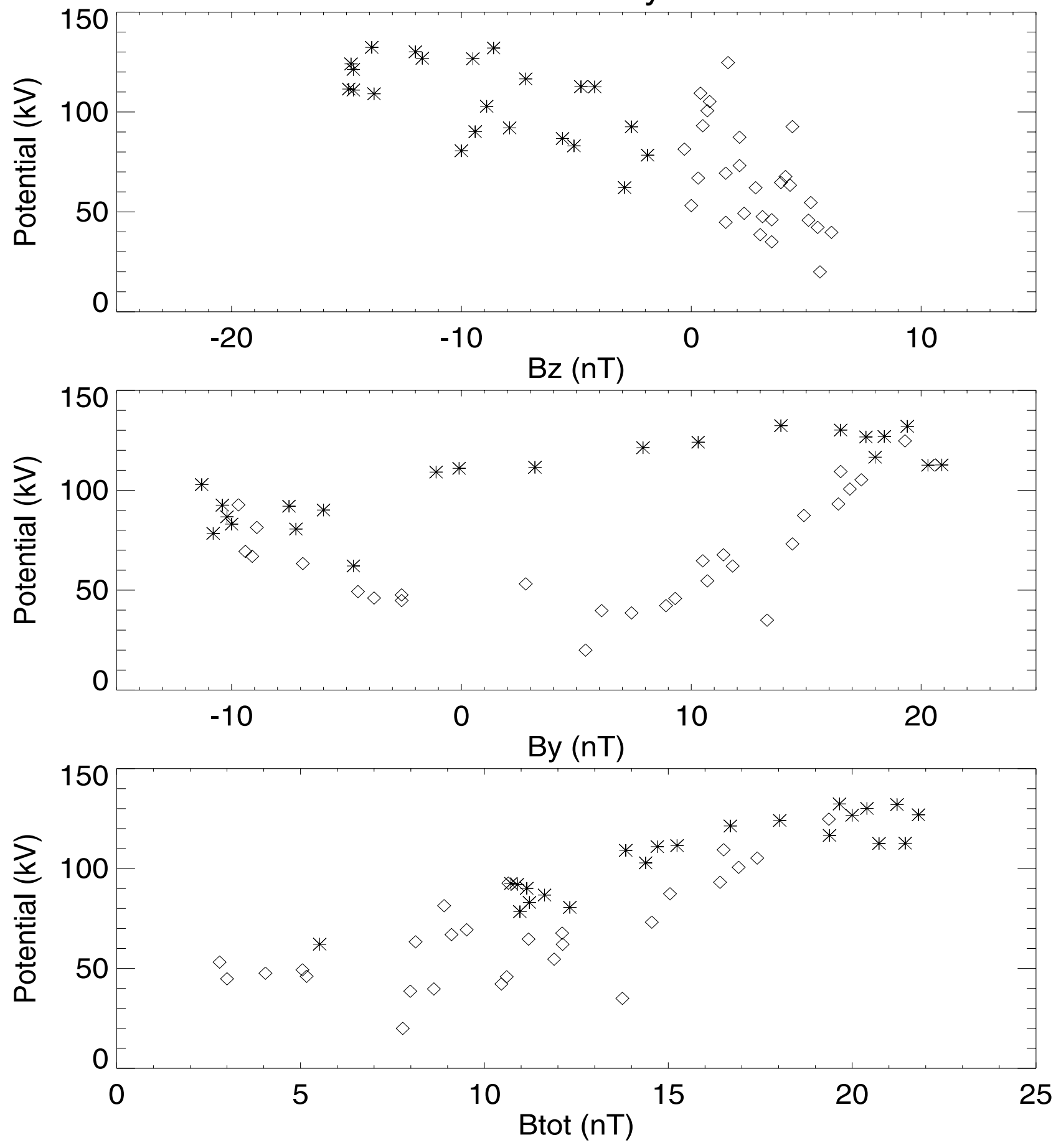

Figure 12. Plots of the ionospheric cross polar cap potential derived from the MHD code versus $B_{z}$ (top), $B_{y}$ (middle), and $\sqrt{B_{z}^{2}+B_{y}^{2}}$ (bottom). The diamonds represent $B_{z} \geq-1$, while the stars represent $B_{z}<-1$.

the ground poorly. Since that study, the ionosphere has been modified. Because the validation in this study is with drift velocities, direct comparisons between the studies can not really be made. It is interesting to note that the RMS errors in this study were far better compared to the data variations.

[36] While direct comparisons between the data and the model are quite useful, there are further ways of validating the general behavior of the code. One of the most important quantities which shows how well the MHD code is doing in general is the cross polar cap potential. By examining this single quantity, one can quickly determine whether the code is systematically underestimating or overestimating the ionospheric (and therefore inner magnetospheric) flow. In addition, some basic properties of the magnetosphere-iono- 
DMSP Only
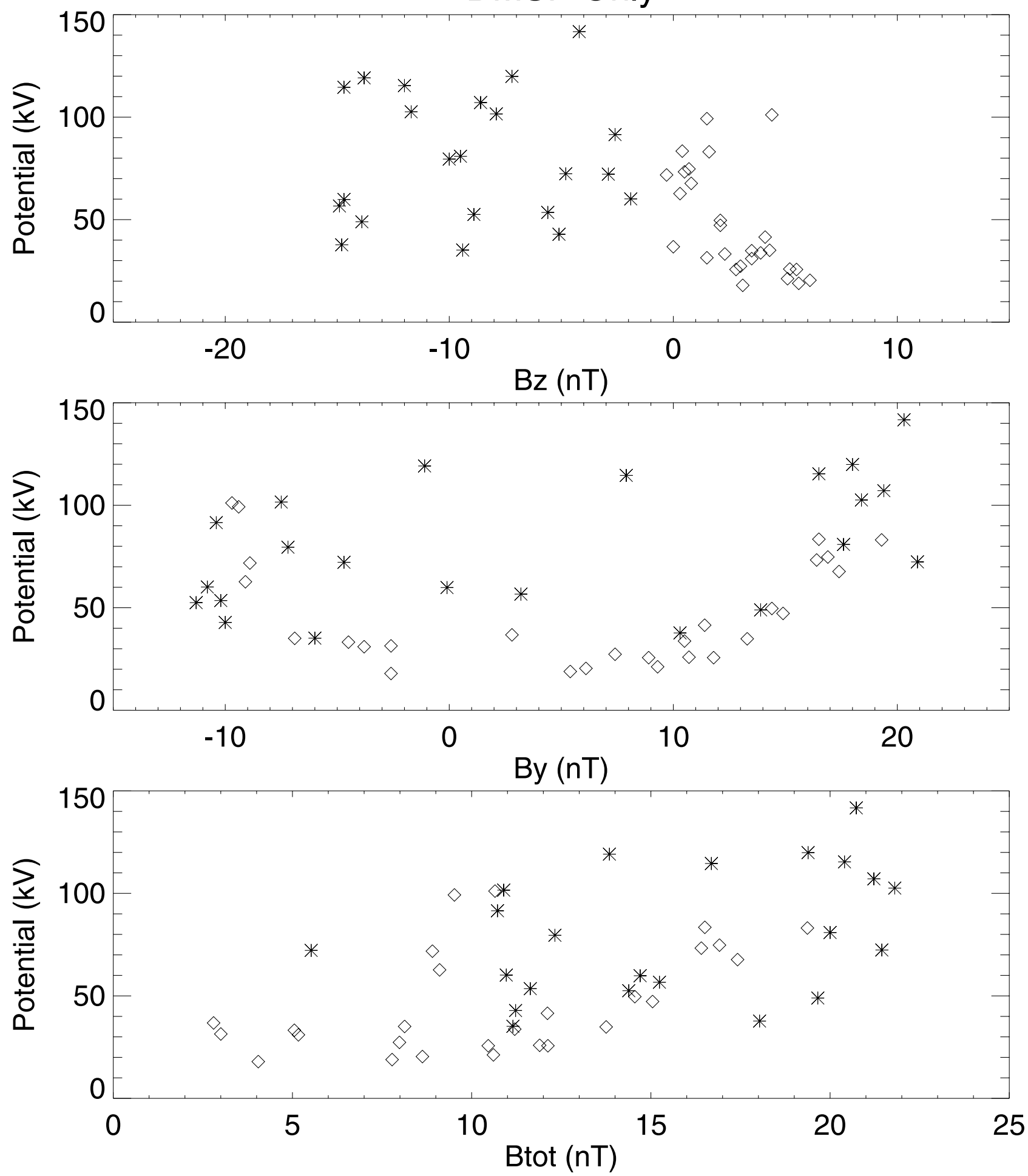

Figure 13. Plots of the DMSP measured cross polar cap potential in the same format as Figure 12.

sphere system may be uncovered by systematic differences between the modeled cross polar cap potential and the actual cross polar cap potential.

[37] For example, as was described above, before the modifications to the ionosphere were made, the code was producing much too large of a cross polar cap potential in the winter hemisphere. Because the electric field is deter- mined by the amount of current into the hemisphere and the amount of conductance in that hemisphere (or vise versa, depending on ones viewpoint), it was either the conductance which was wrong in the code or the strength of the fieldaligned current, or a combination of both. One could then investigate feasibility of the different explanations. In this case, Liou et al. [1997] showed that the auroral brightness 
increases in the winter hemisphere, while the field-aligned currents increase by a factor of 1.5-1.8 in the summer hemisphere [Friis-Christiansen et al., 2002].

[38] As another example, the potentials in the MHD results extended to much lower latitudes than was expected. This was obviously caused by the lack of region-2 currents. In addition, the cross polar cap potential was too high in some cases, which can be caused by a lack of current closure to lower latitudes. A region 2 current system was added to make the ionospheric flows more consistent with the measured DMSP flows.

[39] To investigate whether the MHD code has any further systematic errors in the cross polar cap potential, we compare the modeled and measured potentials. Figures 12 and 13 show plots of the cross polar cap potential of the DMSP data and MHD data against the IMF $B_{z}, B_{y}$, and $\sqrt{B_{y}^{2}+B_{z}^{2}}$. Both figures show that when $B_{z}$ is negative, the cross polar cap potential is larger, which is a well known result. The DMSP data shows that $B_{z}$ can be strongly negative, but the potentials can be small. This is because the DMSP satellite does not always cut through the maximum and minimum in the potential, as is observed in Figures 3, 7, and 10. Therefore, the DMSP potentials are typically thought of as a lower bounds on the cross polar cap potential.

[40] The MHD code shows that the cross polar cap potential is approximately linearly related to $\pm B_{y}$ (independently) when $B_{z}$ is greater than $-1 n T$. On the other hand, when $B_{z}$ is negative, the cross polar cap potential seems to be approximately linearly related to $B_{y}$, with positive $B_{y}$ and negative $B_{z}$ causing the largest cross polar cap potentials. This trend is observed in data derived ionospheric potential models when the IMF magnitude is large [e.g., Ruohoniemi and Greenwald, 1996]. The limited number of data points do no allow definitive conclusions, but the general trends are consistent with both the data and models derived from different data sets.

[41] In addition to the general trend being relatively correct, Figure 12 also shows that the cross polar cap potential may be saturating at high IMF $B_{z}$ values. From this figure, it appears that when $B_{z}$ is below $-8 \mathrm{nT}$, the cross polar cap potential does not increase significantly. This saturation effect has been alluded to in measurements of the ionospheric potential [e.g., Russell et al., 2001], although the value of the saturation potential, and the point at which it starts to saturate are not clear in our study nor in the study of Russell et al. [2001]. Much work is left to do if any definitive conclusion are to be made in this area.

\section{Concluding Remarks}

[42] This study has presented comparisons between simulation results from the University of Michigan BATS-RUS MHD code and ionospheric flows measured by 2 DMSP satellites in both the Northern and Southern Hemispheres. Before the simulations were conducted, the model was tuned using 6 of the approximately 180 passes. The tuning consisted of: (1) adding a region-2 current system, (2) creating a seasonal dependence on the auroral energy input and polar rain, and (3) spatially averaging the field-aligned currents before determining the auroral precipitation pattern. These modifications improved the poor comparisons con- siderably (3 of the 6 passes), while effecting the good passes only a little bit.

[43] Once the tuning was completed, the 3 events were run. It is shown that the results appear to agree with the DMSP data almost as well as the Weimer [1996] patterns, although the statistical model is better than the MHD code on average. The April 1999 time period was run in both local time stepping mode with high resolution and timeaccurate mode with lower resolution, while the other events were run only in the high resolution local time stepping mode. It was shown that the high resolution mode does better for slowly varying IMF periods, but when the IMF is changing rapidly, the lower resolution, time-accurate mode is better. This was due to the fact that the convection velocity was most likely changing in time as the DMSP satellite measured the flows (while the local time stepping velocities do not change in time). It was also shown that the model tendencies in the behavior of the cross polar cap potential reproduces data-derived models such as Ruohoniemi and Greenwald [1996].

[44] The discussion above assumes that a valid method for validating the MHD code is the comparison of ionospheric data to the ionospheric results from the code. While this may be enlightening, it does not prove how well the code is modeling the magnetosphere, only the ionosphere. It may not even do that well.

[45] For example, the above discussion focuses only on the flow velocity of the ionosphere. The MHD code outputs the field-aligned currents into the ionosphere, then using a conductance pattern, the electric potential, electric field, and flow velocities are determined. If the MHD code output FACs which were a factor of 10 to large, the conductance could be modified such that the electric field, and therefore the flow velocities were correct. Therefore, the MHD solution would be wrong and the ionosphere (i.e., conductances) would be wrong, but the answer would be correct.

[46] A better metric may be one which is taken from the magnetosphere itself. This would test the validity of the MHD results in the MHD modeled region of space, instead of a projection of the MHD results outside of the modeled region. We suggest a number of metrics which may be more meaningful: (1) the location of the magnetopause for given inbound and outbound flights for various satellites such as IMP 8, Geotail, Interball, Wind, etc.; (2) location of the cusp as determined by the Polar satellite; (3) bulk flow velocities in the tail measured by various satellites (such as hourly averages, or hourly averages with standard deviations to show the variations); and (4) magnetic field orientation and magnitude for given geosynchronous satellites, such as GOES. While each of these validations may have their weaknesses, it would be better to compare magnetospheric data with magnetospheric models, instead of ionospheric data with magnetospheric models.

[47] If we backtrack and assume that the ionospheric conductances are more or less correct in the simulation, then the RMS would only quantify the degree of error within the model, but not whether the error is systematic in any manner. For example, if the modeled flow velocities were consistently $1 / 3$ below the DMSP velocities, the RMS error would be the same as if it were $1 / 3$ above the measurements. Also, if the errors were completely random, but on average the deviation was $1 / 3$ away from the DMSP 
velocities, the RMS would be approximately the same. But one would not be able to tell the difference just by examining the RMS errors.

[48] On the other hand, if a model consistently received very low RMS errors or very large RMS errors, it would be clear that the model is either quite capable of nearly reproducing the data, or that the model had fundamental flaws, respectively. It is the RMS errors which are not poor nor great which are difficult to interpret.

[49] We suggest that using some other form of quantifying the systematic errors within the code may be more meaningful for the community than RMS errors such as comparing the general behavior of the flow velocities or cross polar cap potentials with strength of the IMF, or comparing the location and sharpness of the flow reversals within the results. By using these type of criteria, true understanding of the systematic errors within a model can highlighted, shared with the community, and eventually rectified.

[50] Acknowledgments. The research for this manuscript was supported by Department of Defense Multidisciplinary University Research Initiative grant F49620-01-1-0359, National Science Foundation grants ATM-9980078 and ACI-9876943, and National Aeronautics and Space Administration grant NAG5-9406.

[51] Janet G. Luhmann thanks Marc R. Hairston and another referee for their assistance in evaluating this paper.

\section{References}

Fedder, J. A., S. P. Slinker, and J. G. Lyon, A comparison of global numerical simulation results to data for the January 27-28, 1992, Geospace Environment Modeling challenge event, J. Geophys. Res., 103, 14,799, 1998.

Friis-Christiansen, E., V. O. Papitashvili, and T. Neubert, Seasonal variations of high-latitude field-aligned current systems inferred from Ørsted and Magsat observations, J. Geophys. Res., 107(A2), doi:10.1029/ 2001JA900104, 2002.

Gombosi, T. I., K. G. Powell, and B. van Leer, Comment on "Modeling the magnetosphere for northward interplanetary magnetic field: Effects of electrical resistivity" by J. Raeder, J. Geophys. Res., 105, 13,141, 2000.

Groth, C. P. T., D. L. DeZeeuw, T. I. Gombosi, and K. G. Powell, Global three-dimensional MHD simulation of a space weather event: CME formation, interplanetary propagation, and interaction with the magnetosphere, J. Geophys. Res., 105, 25,053, 2000.

Hairston, M. R., and R. A. Heelis, Response time of the polar ionospheric convection pattern to changes in the north-south direction of the IMF, Geophys. Res. Lett., 22, 631, 1995.

Liou, K., P. T. Newell, C.-I. Meng, M. Brittnacher, and G. Parks, Synoptic auroral distribution: A survey using Polar ultraviolet imagery, J. Geophys. Res., 102, 27,197, 1997.

Powell, K. G., P. L. Roe, T. J. Linde, T. I. Gombosi, and D. L. De Zeeuw, A solution-adaptive upwind scheme for ideal magnetohydrodynamics, $J$. Comput. Phys., 154, 284, 1999.

Raeder, J., Modeling the magnetosphere for northward IMF: Effects of electrical resistivity, J. Geophys. Res., 104, 17,357, 1999.

Raeder, J., Reply, J. Geophys. Res., 105, 13,149, 2000.

Raeder, J., J. Berchem, M. Ashour-Abdalla, L. A. Frank, W. R. Paterson, K.

L. Ackerson, S. Kokubun, T. Yamamoto, and J. A. Slavin, Boundary layer formation in the magnetotail: Geotail observations and comparisons with a global MHD simulation, Geophys. Res. Lett., 24, 951, 1997.

Raeder, J., J. Berchem, and M. Ashour-Abdalla, The Geospace Environment Modeling Grand Challenge: Results from a Global Geospace Circulation Model, J. Geophys. Res., 103, 14,787, 1998.

Rich, F. J., and M. Hairston, Large-scale convection patterns observed by DMSP, J. Geophys. Res., 99, 3827, 1994.

Richmond, A. D., and Y. Kamide, Mapping electrodynamic features of the high-latitude ionosphere from localized observations: Technique, J. Geophys. Res., 93, 5741, 1988.

Ridley, A. J., Estimation of the uncertainty in timing the relationship between magnetospheric and solar wind processes, J. Atmos. Sol. Terr. Phys., 62, 757, 2000.

Ridley, A. J., D. L. De Zeeuw, T. I. Gombosi, and K. G. Powell, Using steady state MHD results to predict the global state of the magnetosphereionosphere system, J. Geophys. Res., 106, 30,067, 2001.

Ruohoniemi, J. M., and R. A. Greenwald, Statistical patterns of high-latitude convection obtained from Goose Bay HF radar observations, J. Geophys. Res., 101, 21,743, 1996.

Russell, C. T., J. G. Luhmann, and G. Lu, Nonlinear response of the polar ionosphere to large values of the interplanetary magnetic field, J. Geophys. Res., 106, 18,495, 2001.

Song, P., D. L. De Zeeuw, T. I. Gombosi, C. P. T. Groth, and K. G. Powell, A numerical study of solar wind-magnetosphere interaction for northward interplanetary magnetic field, J. Geophys. Res., 104, 28,361, 1999.

Weimer, D. R., A flexible, IMF-dependent model of high-latitude electric potential having "space weather" applications, Geophys. Res. Lett., 23, 2549, 1996 .

D. L. De Zeeuw, T. I. Gombosi, K. C. Hansen, A. J. Ridley, and G. Tóth, Space Physics Research Laboratory, Department of Atmospheric, Oceanic, and Space Physics, University of Michigan, Ann Arbor, MI 48109-2143, USA. (ridley@umich.edu)

K. G. Powell, W. M. Keck Foundation Computational Fluid Dynamics Laboratory, Department of Aerospace Engineering, University of Michigan, Ann Arbor, MI 48109-2140, USA. 